\title{
A Survey on Opportunistic Routing
}

\author{
Saleh A. Khawatreh ${ }^{1}$, Mustafa Abdullah ${ }^{2}$, Enas N. Alzubi ${ }^{3}$ \\ Computer Engineering Dept., Faculty of Engineering, Al-Ahliyya Amman University, Amman-Jordan ${ }^{1,3}$ \\ Civil Engineering Dept., Faculty of Engineering, Al-Ahliyya Amman University, Amman- Jordan ${ }^{2}$
}

\begin{abstract}
Opportunistic Routing (OR) is attracted much in the research field of multi-hop wireless networks because it is different from traditional routing protocols [such as: Distance Vector (DV) and Link State (LS)], that it needs a lightweight protocol which is strong source routing basis]. In this paper, the development of opportunistic routing (OR) is presented, starting from the Selection Diversity Forwarding (SDF), Extended Opportunistic (ExOR), Proactive Source Routing (PSR), Cooperative Opportunistic Routing Scheme in Mobile AD Hoc Networks(CORMAN), and the Zone-based Proactive Source Routing(ZPSR). The simulation tests using Network Simulator 2 (ns2) show the effectiveness of Opportunistic Routing protocols among various terms including control overhead, Packet Delivery Ratio (PDR), throughput, and end-to-end delay.
\end{abstract}

Keywords-Opportunistic routing; PSR; ExOR; proactive routing; source routing; tree-based routing; lightweight routing; wireless networks; ad hoc networks

\section{INTRODUCTION}

Mobile Ad Hoc Networks (MANET) is a challenging field due to its infrastructure which has less nature and time varying medium that effected by wireless medium propagation and multi-path fading, where nodes are free moving and selfconfiguring wireless communication links among them. There is no direct link to reach a node, so if any node wants to send a message to other node which it may not be its neighbor so in this case a multi hop communication is needed. In addition, wireless networks are soft links, that means, the link status change among the time, so the transmission power and the routing decisions are effected according to that, finally, Ad hoc networks has many applications such as: battlefield, health care, security, industry, environmental monitoring, and emergency operations in rescue and search. So many researches have been published since 1980s [1]. The most challenges in MANETs include end-to-end delay, security, and real-time multimedia sending.

Numerous routing protocols in the network layer have been created where everyone has its advantages and applications. Two distinct functions of the network layer are: routing and forwarding, forwarding is the process where nodes regulate the packets transfer from one link to another. Routing is a different process where nodes need to determine which path to select among transferring the packets to the destination and this need a control inputs, despite the huge efforts in data routing in MANETs, data forwarding is less challenging and follows the mechanism in Internet Protocol (IP), which was designed for wired networks. So the major goal in the research is to make wireless links as good as wired ones.
Opportunistic Routing (OR) has become the most attractive paradigm in wireless networks, due to its feature to develop the performance of wireless ad hoc networks. It takes advantage of broadcast characteristics and the spatial diversity, by packet transmit overhearing and the coordinating between relays nodes, OR sets a set of candidate nodes to act like a forwarding nodes to transmit packets to the destination. This dynamic sets of relays increase the performance of the network and the results improve that it increases the capacity, throughput, and decrease end-to-end delay, power consumption and control overhead [2]. In other words, OR is a routing techniques where a node broadcasts packets and the nodes which receive it correctly take the part of the data forwarding until the packets reach the destination, according to that, any node can participate in data forwarding based on the reception status of the packets, which differs from the traditional data routing IP where each intermediate node must check its routing table to take a decision which path to use.

Many categories of routing protocols are proposed in MANETs, the first category is proactive protocols (or named table-driven), in these protocols, every node has an information about the network topology, the nodes periodically flood its information about the link status to its all neighbors, so after certain time, all the nodes will have the whole topology of the network, the most popular protocols which belong to this category include Optimized Link State Routing (OLSR) [3] and Destination Sequenced Distance Vector(DSDV) [4]. The second category is reactive protocols (named as on-demand), in this protocols, nodes not always have routing information, instead that, routing information is constructed only when needed, and some examples include: Dynamic Source Routing (DSR) [5] and Ad-hoc On-demand Distance Vector (AODV) [6]. The third category is the hybrid routing, where nodes have entire routing information to some destinations and calculates routing information to others, it is a mixture between the first two ones, these routing protocols can be categorized into Link State (LS) and Distance Vector (DV), based on their algorithms, where in LS routing, every node exchanges information about itself among all nodes in the network where nodes in DV routing algorithm exchange the cost to each destination among neighbors.

To support opportunistic forwarding in MANETs, we need a routing facility that can offer source routing like DSR and gives robust information about the links like OLSR, but these protocols cannot be used due to their overhead, so a new routing protocol is proposed which is PSR that is described later. 
In this paper, we present a survey of the improvements on OR starting with the Selective Diversity Forwarding (SDF), Extended-Opportunistic Routing (ExOR), Proactive Source Routing (PSR), Cooperative Opportunistic Routing Scheme in Mobile AD Hoc Networks (CORMAN), and the Zone-based Proactive Source Routing (ZPSR).

The remainder of the paper is organized as the following: Section II presents the Opportunistic routing protocols, Section III includes computer simulation for PSR and CORMAN, and finally, Section IV concludes the paper.

\section{OPPORTUNISTIC ROUTING PROTOCOLS}

In this section, different Opportunistic Routing (OR) protocols are presented, one of the first researches in OR proposed the Selective Diversity Forwarding (SDF) [7] where the transmitter chooses the best relays from multiple receivers that correctly receive its packets, then these selected relays take part of the data forwarding. However, it did not participate in real life due to its high control overhead. This inspired the researchers and led to propose an extension to OR which was ExOR by Morris and Biswas [8], they proposed anew cross-layer solution between the link and network layers. They take advantages of OR and DFS. In ExOR, the transmitter selects a set of forwarders which forward the data to the distinct destination according to the closeness to the destination, according to medium-access-control (MAC) sub layer it controls the contention and manage the data forwarding. To enhance the ExOR in wireless networks we need to know the nodes IDs and the topology of the networks and this needs to participate source routing protocol and link state routing protocol, and that results in a huge overhead, so a new routing protocol has proposed, a lightweight Proactive Source Routing (PSR) [9] where each node calculates a Breadth-First Spanning Tree (BFST) rooted at itself and includes all the nodes in the network. A Novel Cooperative Opportunistic Routing Scheme in Mobile Ad Hoc Networks (CORMAN) is presented in [10]. Finally, the new Zone-based Proactive Source Routing (ZPSR) [11] is proposed, where the network is divided into clusters and PSR is applied in the inter cluster, and outside, a Zone-based Routing Protocol (ZPR) [12] is used, and that improves the network performance. Here are the details:

\section{A. Selection Diversity Forwarding (SDF)}

In MANETs traditional routing schema is based on the shortest path to destination algorithms, such as, DV, LS. Many problems of these protocols include the instantaneous propagation and the exploitation less of the broadcast nature.

Larsson in his paper proposed SDF, it use the model of selection diversity in the framework in routing, it categorized as non-deterministic routing, and this is the first study of SDF according to fading and non-fading medium status. This method has three parts, described below:

1) Network model: Nodes broadcast the packets as slotted ALOHA, the nodes are half duplex type, and assumed distributed in uniform way on a square surface that density is $\lambda$ and the direction of the transmission is controlled by transmitting terminal, nodes can receive these packets according to the following equation:

$P i * \frac{G(R o)}{N}=S N R \min$

Where presents the transmitting power of node $i, N$ is the receiving power, $R o$ is the distance between the nodes, $G(R o)$ is the gain of the channel, and the SNRmin is the minimum signal to noise ratio that is accepted to successive reception.

Two scenarios were studied, the first when one node is transmitting and the others are receiving, and this is called as heterogonous traffic. On the other hand, a heavy loaded network is considered, and each node has one packet at least to send, and this system is considered as homogenous model.

2) Capture model: A capture model is proposed with the probability of the reception according to the following:

$P k=\left\{\begin{array}{c}1, \quad \text { if } \frac{\text { PiGik }}{N+\sum j \neq i, k \text { Pj.Gjk }} \geq \text { CIRmin } \\ 0, \text { otherwise }\end{array}\right.$

When node $\mathrm{i}$ sends a packet to node $\mathrm{k}$, many interfered nodes denoted by j. Gik and Gjk are link gains and CIRmin represents the minimum required signal carrier to interference ratio. The gain of the channel is assumed to be constant.

3) Propagation models: Here two channel models are experienced: a non-fading and a Rayleigh channel.

In the first model, the gain of the channel is based to the distance $r$ as in the below equation:

$G(r)=$ Constant.$r^{-a}$

In the second model, the Rayleigh fading is proposed uncorrelated from slot time and illustrated as the following:

$f G(\gamma, R)=\frac{1}{G(r)} e^{\frac{-\gamma}{G(r)}}$

Where $\gamma$ represents a random variable of the gain.

The operation of SDF is shown in Fig. 1, according to it, the node broadcast the packets, then many nodes receive it and send acknowledgements sequentially to the sender then the sender sends a forwarding order then an acknowledgements are sent back.

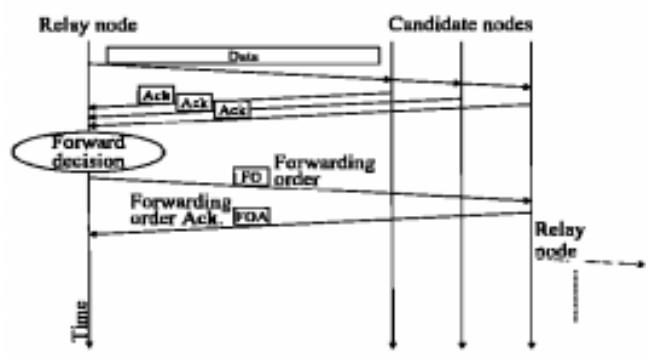

Fig. 1. Channel Access Mechanism in SDF. 
It is fact that this protocol is not assumed to operate solely, it needs a protocol that provides the direction to the destination. One of the simplest connections is using Bellman Ford Routing [12], in which nodes select the relay nodes according to the cost to the destination. Other models can be used as power control; link rate could also be used to increase the performance. However, it increases the complexity of the design. Finally, SDF increased the network performance compared with the single path routing such as MFP and NFP. But the major drawbacks of this protocol are its overhead and cannot be used in sole model.

\section{B. Extended-Opportunistic Routing (ExOR)}

ExOR is an integrated extension and MAC protocol which improves the performance of big unicast data transmissions in MANETs. Here every hop is choosing after the transmission for this hop, and therefore, the paths are determined according to the real transmissions.

ExOR model has three challenges. The nodes which receive the packets need to be agreed on the nodes identities and pick one forwarder. This agreement should not cause a large overhead, so it need to be a lightweight, however, it should be robust to ensure that all the packets are received and don't need to retransmissions. Finally, the forwarder must be chosen as the closest to the destination.

A simple ExOR can be studied according to Fig. 2. In which a simple network is presented, suppose the source node wants to send packets to the destination. First, the source broadcasts the packets, the nodes then uses a protocol to know which nodes in the sub-set. The closest node to the destination is selected as a forwarder; the process is repeated until the destination receives the packets, back to Fig. 2. The probability to reach an intermediate node is $10 \%$, and the probability to reach the destination from these intermediate is $100 \%$. In traditional routing if the source node cannot transmit to the specific intermediate node then it must to retransmit until it reaches even though it reached other ones.

Fig. 3 shows another advantage of ExOR, according to this figure, a chain of nodes is considered, when the source wants to send a packet to the destination, in the traditional routing, the source first selects a certain path for example, src-B-D-dst. If node B didn't receive the packets, then the source must to retransmit the packets until node $B$ receive it, however, other nodes received the packet where it may be near to node $\mathrm{B}$ (before or after it). In ExOR, the source does not need to retransmit the packets; any intermediate node can forward the packets.

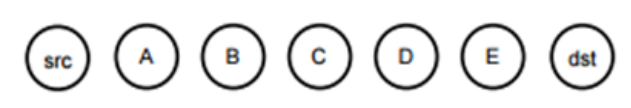

Fig. 3. A Chain of Network Nodes Example.

The ExOR design has four challenges. First, the nodes should have an agreement, in which it contains the sub-set of the nodes that received each packet, since this agreement is a control messages it must be a lightweight control overhead but robust simultaneously. Second, the closest node to the destination must be chosen as a forwarder node. Third, if the network is large and dense, there will be a delay if many nodes are selected as participants, so it is better to choose the most effective ones only. Finally, ExOR should avoid simultaneous transmissions to avoid collisions.

To eliminate the control overhead, ExOR uses a patch of packets every transmission, so the agreement control messages will decrease to a significant ratio.

1) Node state: Every ExOR node maintains the state for every batch of the packets which is participating in the transmission, according to the node's presence in the batch's forwarder list. Nodes start keeping the state of the nodes after receiving the first packet. Then it stores the received packets in its batch.

The forwarder list includes a copy of the prioritized nodes list, which copied from a packet in the packet buffer. For a batch, all nodes contain the same forwarder list, which generated by the source.

The forwarding timer determines the time at when the node must forward its packets, and this must be set far from the receiving.

The transmission stalker tracks the measured rate at when the current node is sending, based on the expected packets numbers, which has left to send later. The nodes use this data to set the forwarding timer. The batch map shows, for every packet in the batch, the node which have the highest-priority to let it received a copy of the packet.

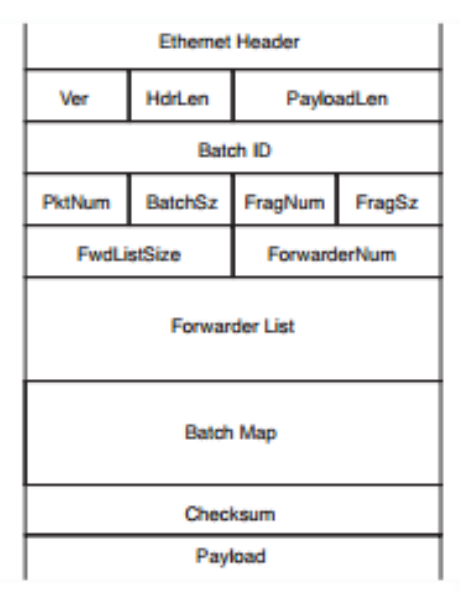

Fig. 4. ExOR Packet Header.

Fig. 2. Network Nodes Example. 
2) Packet format: Fig. 4 presents the ExOR's packet header. It follows the Ethernet header, and the data follows the Ethernet header. All ExOR packets are broadcasted. The Ver field in Fig. 4 references to the ExOR version, this is used to future changes. The HdrLen field presents the size of the ExOR header and Payload Len presents the size of the payload. The BatchID field references to the batch the packets belong to. The PktNum represents the packet's offset in this batch. This offset represents the map of the batch entries for each packet. The BatchSz corresponds to the number of packets in each batch. FragSz presents the size of the current fragment (in packets), and FragNum presents current packet's offset of the fragment. The FwdListSize field presents the number of forwarders which are in the list. the forwarder num indicates the sender's offset among the list. Finally, The Batch Map is a batch map of the sending's node copy; to save space, every entry is an indicator to the forwarder List.

3) Batch preparation: The source collects a batch of packets which all estimated to the same destination. It picks a new batch ID and picks a list of forwarders. The source only has the all packets and that is illustrated in the batch map. Finally, the packets are broadcasted from source.

4) Forwarder list: The source indicates the forwarder list as a priority list accorded to the predictable cost of sending a packet between the nodes in the list until it reaches the destination. The cost parameter is the number of hops required to move a packet by the traditional routing from a node to a destination, including hops and retransmissions.

The source picks the forwarder list using the information about the network. The source gets this information via a periodic link-state message. ExOR is insensitive to fault or out-of-date bulks, because a packet's path is specified by status of the time of transmission.

5) Packet reception: Each node checks the header of each successful packet. If the list contains the node, the node put the packet into the buffer to a corresponding batch. For every item in the batch map included in the packet, then the node compares the item with the items in the batch map, and exchanges the older if the packet's item which is the highest priority node.

6) Scheduling transmissions: ExOR tries to manage when the nodes send their fragments so at a time only one node sends its packet. This manner allows the highest-priority nodes to send first. This scheduling avoids collisions.

7) Completion: When a node's batch map points that over $90 \%$ of the batch is received by the highest priority nodes, the node sends nothing. The last packets in the batch will be the most expensive to send, because it would need all the overhead of the transmission management, although, the overhead will be divided among few packets, and if the fragments are small, there is a greater probability that nodes will suppose their timers incorrect and then collide.
Because ExOR warranties to receive $90 \%$ of a batch, the destination requests the packets which remained by a traditional routing. The destination sends the batch map back to the source, then sends these packets by traditional routing, using link-level information to obtain a reliable delivery.

The major drawbacks of ExOR are it doesn't support reliable delivery, and there are many questions that are not answered; how to choose the candidate nodes? How many and which neighbors to select as forwarder lists?

\section{Proactive Source Routing (PSR)}

PSR supplies each node with a breadth-first spanning tree (BFST) about the whole network started in itself. To support that, nodes broadcast the tree structure every interval, to their neighbors, anode expand and refresh its information about the network by structure a longer and more recent BFST. This Information then will be flooded to its neighbors in the next period. When a neighbor is become lost, a technique is followed to remove its pertinent information from the topology of the detecting node.

1) Route update: Because of its proactive nature, all nodes exchange these BFST, with its neighbors and a star paradigm is obtained denoted as $\mathrm{Sv} . \mathrm{N}[\mathrm{v}]$ presents the close neighbors and $\mathrm{N}(\mathrm{v})$ presents the open neighbors. And this technique uses the following equation:

$G v=S v \cup \cup_{u N(v)}(T u-v)$

In the above equation if $\mathrm{T}-\mathrm{x}=\mathrm{T}$, we conclude that $\mathrm{x}$ is not in $\mathrm{T}$, and if $\mathrm{T}-\mathrm{x}=0$, so that $\mathrm{T}$ is $\mathrm{x}$.

2) Neighborhood trimming: These periodic broadcast flooding as a model as "hello" messages when a neighbor is considered lost, its tree to the network connectivity must be removed; this is named as neighbor trimming. Assume node $v$. The neighbor trimming technique is considered at $v$ about node $u$ that is a neighbor of $v$ by one of the following ways:

a) Routing updates or data packet has not been received from node $u$ for a specific period of time.

b) A transmission to node $u$ has not succeeded, as denoted in the link layer.

Node $v$ reacts by:

- Refreshing $\mathrm{N}(\mathrm{v})$ by $\mathrm{N}(\mathrm{v})-\{\mathrm{u}\}$.

- then, computing the union paradigm with the information of $u$ removed, i.e.,

$\mathrm{G} v=S v \cup(T w-v)$

3) Finally, constructing BFST $T_{v}$. and is not flood immediately to avoid extra messaging. This updating manner at $\mathrm{v}$ is ensuring avidness of sending packets by lost neighbors. So, many neighbors trimming techniques may be constructed in one period.

4) Streamlined differential update: Flooding route updates as hello messages in PSR, helps to replace the "full dump" 
routing, with "differential updates." and to send these full updated messages less than those short messages. In PSR, two methods are constructed to decrease the overhead; first, they used a compressed tree representation in both updated messages to decrease the size of it. Second, each node tries to keep a refreshed BFST in every change of the network so the differential updates are shorter.

5) Compressed tree representation: In this method we use binary tree representation such as shown below for the binary tree show in Fig. 5. A10B11C11D10E00F00G11H00I00

Stable BFST, by calculating the following equation:

$(T v-u) \bigcup_{w N(v)}(T w-v)$

\section{A Cooperative Opportunistic Routing Scheme in Mobile} AD Hoc Networks (CORMAN)

CORMAN is a pure network layer protocol based on opportunistic routing in MANETs. Its node communication mechanism is based on ExOR. First it is better to highlight the objectives and the challenges, and these objectives are: It extends the applicability of ExOR, and reduces the overhead of ExOR by choosing a shorter list of forwarders than in ExOR. The challenges are:

1) Overhead in calculating the route.

2) Adaptation of the forwarder list.

3) Robustness versus link variations.

CORMAN routes the packets in a batch manner similar as in ExOR. Data packets are collected into batches. And all packets in the batch have the same forwarder list before they transfer from the source. To boost CORMAN, they have a Proactive Source Routing (PSR) that provides every node with a complete routing knowledge to all the nodes in the network. So, the forwarding list includes the nodes identities on the path starting from the source until the destination. As long as, the packets forwarded among the nodes, the nodes which listed as forwarders can notice a change of the topology. This is named as large-scale live updates. And, in addition to that, CORMAN allows other nodes which are not considered as forwarders to retransmit packets if there some missing or fault, and this is referred to as small-scale retransmission.

Therefore, CORMAN has the following modules. Every module solves one of its challenges.

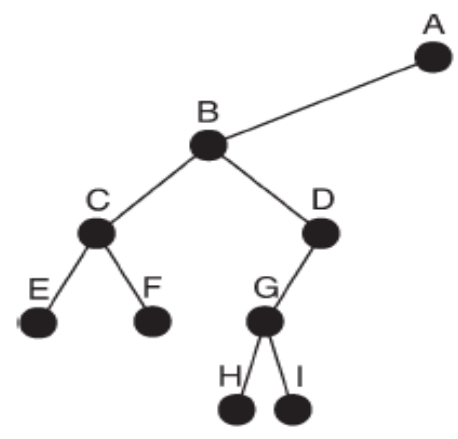

Fig. 5. Example of a Binary Tree.
1) Proactive source routing (PSR) used as a background and nodes periodically refresh its knowledge about network structure, by exchanging messages among the neighbors.

2) Large scale live updates. When the packet received in a forwarding node, this node can change the forwarder list, due to, its better knowledge about the destination as shown in Fig. 6.

3) Small scale retransmission. Because CORMAN uses a short forwarder list, it needs a mechanism to ensure the robustness among the transmission, to obtain this, the nodes which are not consider as a forwarder control the transmissions and retransmit the missed ones as shown in Fig. 7.

CORMAN has the following interesting ways to research:

1) It is better to further test CORMAN, such as, comparing it with ExOR model and IP forwarding.

2) The symmetry of multiple small-scale

3) Retransmissions could be obtained with better methods than RSSI.

4) Nodes in CORMAN transmit data packets in fragments. Among multiple nodes, it could allow nodes in different segments to operate at the same time.

\section{E. The Zone-based Proactive Source Routing (ZPSR)}

This is a hybrid protocol that combines the advantages of both PSR and ZRP. The basic idea of this protocol is to group the nodes in clusters and every cluster has a cluster head that is responsible of communicating with the outside world and to collect the data from the nodes members and send it outside. In the cluster the nodes perform the PSR, and in the outside the ZRP is used, that way gives the improvements to the performance.

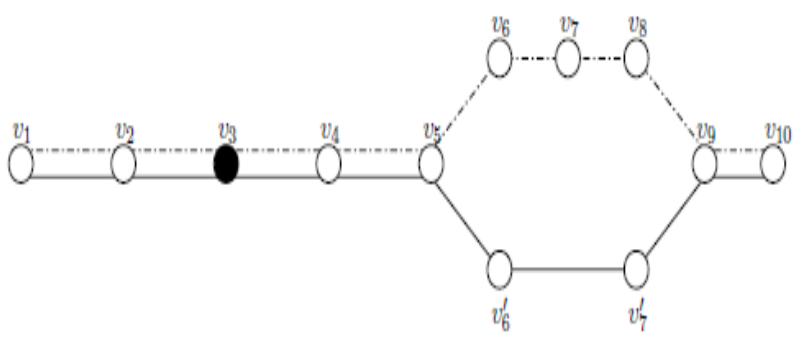

Fig. 6. Route updaing Example.

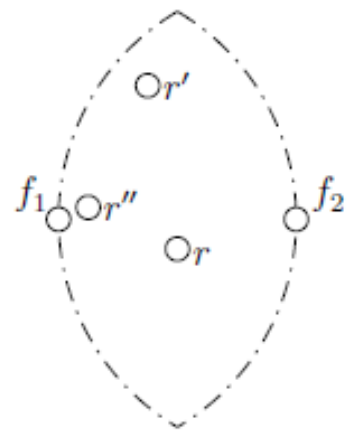

Fig. 7. Retransmission Region Example. 


\section{Performance Evaluation of CORMAN}

In this section, the performance of CORMAN is presented by doing computer simulation using Network Simulator ns-2 (version 2.34). It is compared with AODV in networks with different densities and mobility of the nodes. Then the performance explorations of these results are discussed. The PDR is plotted versus the network dimensions (Fig. 8) to show the performance relation between the CORMAN and AODV. The average delay is plotted versus the network dimensions (Fig. 9) to show the performance relation between the CORMAN and AODV. The delay jitter is plotted versus the network dimension (Fig. 10) to show the performance relation between the CORMAN and AODV.

The PDR is plotted versus the nodes mobility (Fig. 11) to show the performance relation between the CORMAN and AODV.

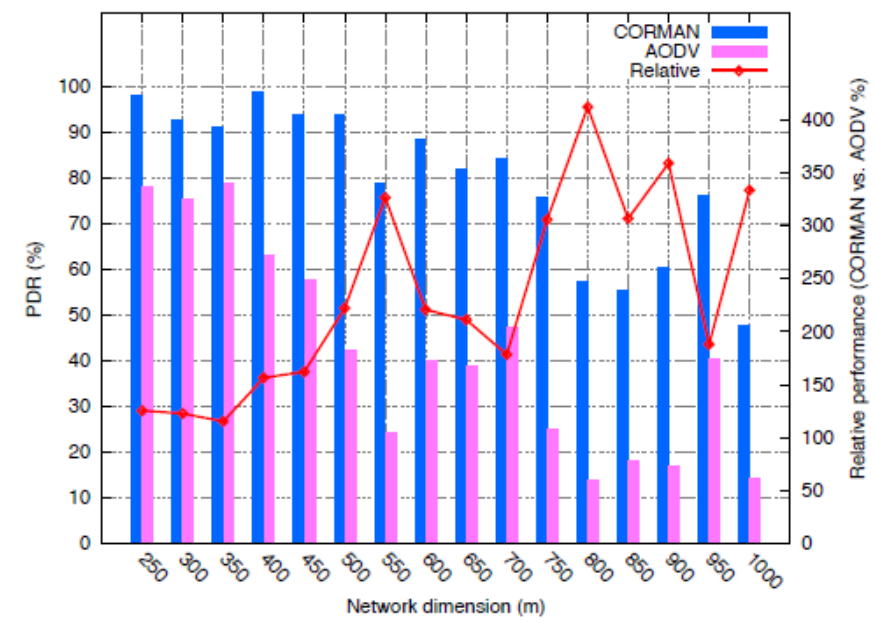

Fig. 8. PRD vs. Network Dimensions.

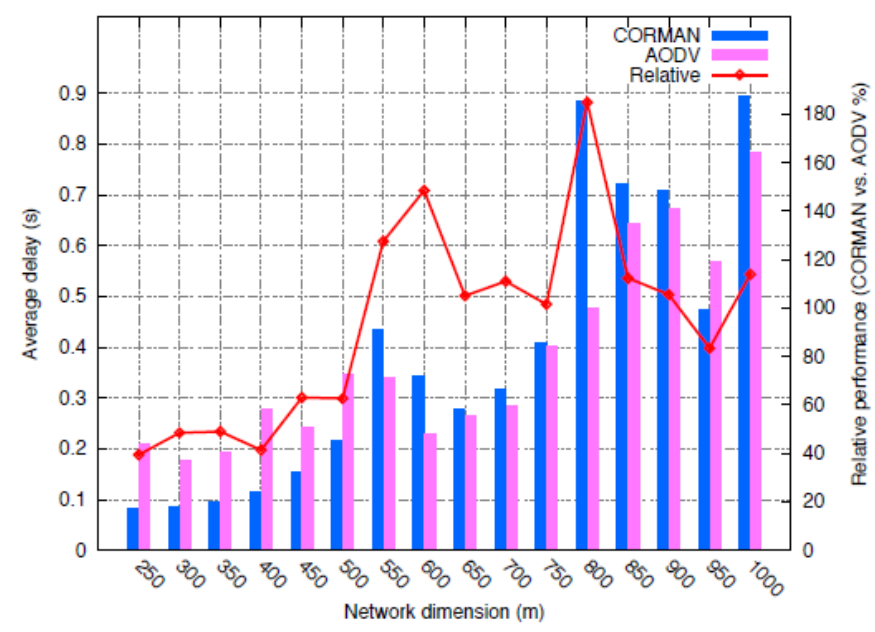

Fig. 9. Delay vs. Network Dimensions.

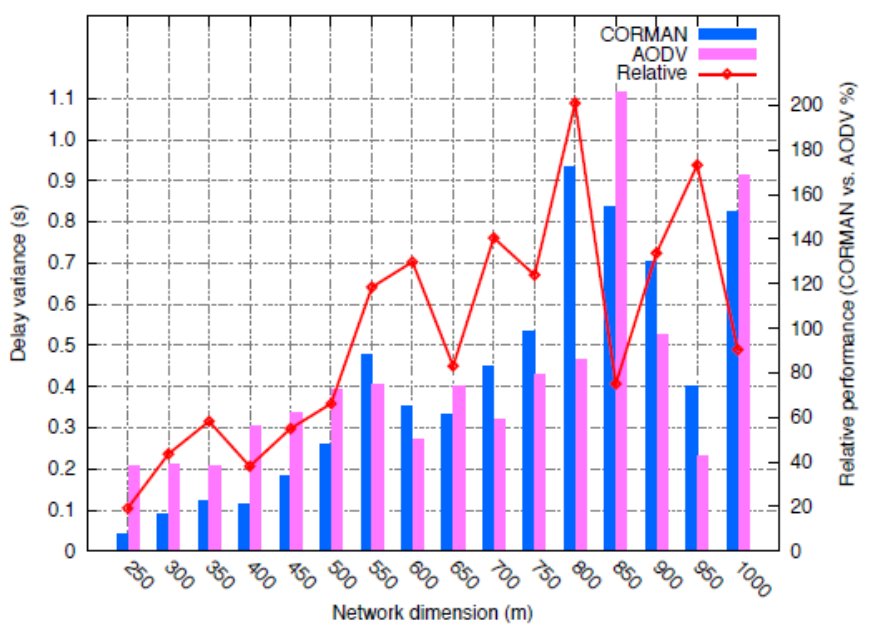

Fig. 10. Delay Jitter vs. Network Dimensions.

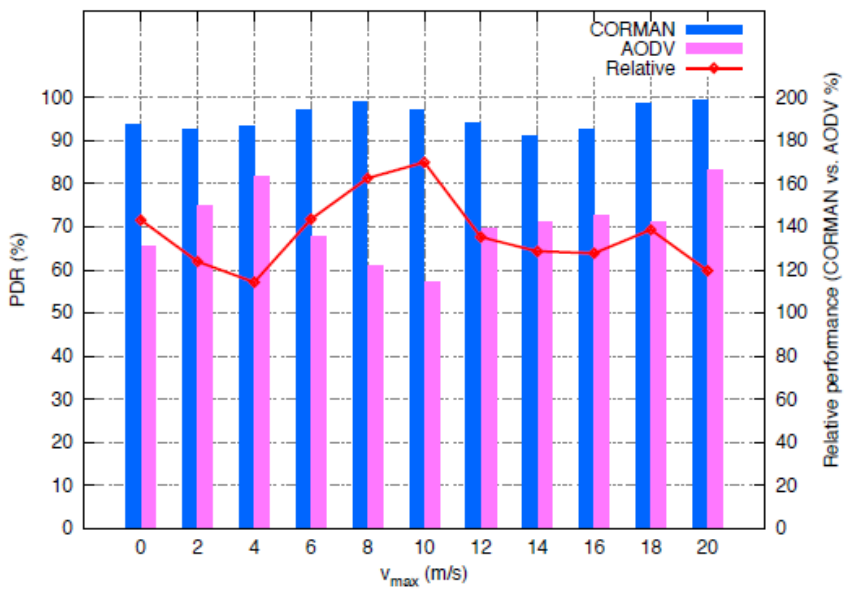

Fig. 11. PRD vs. Nodes Mobility.

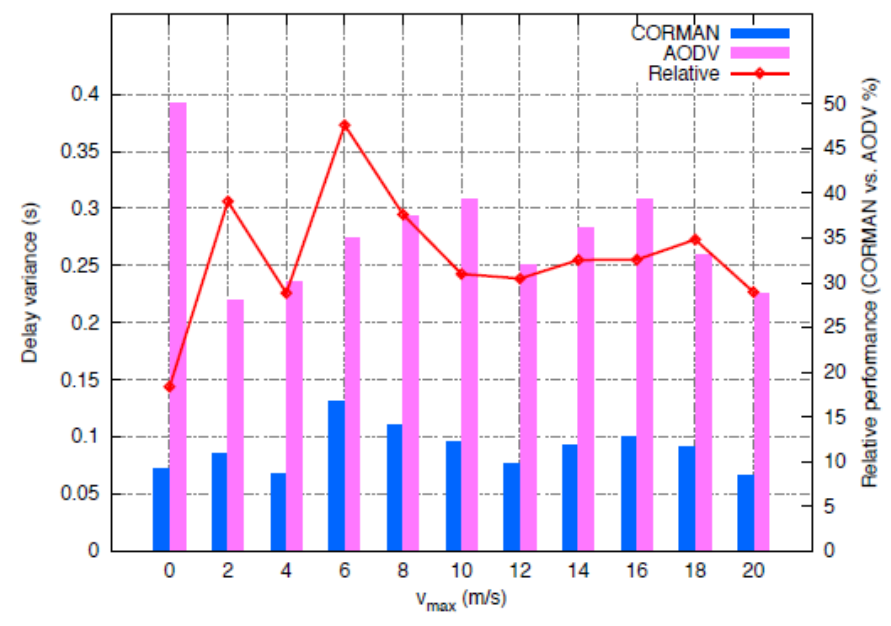

Fig. 12. Delay vs. Nodes Mobility. 


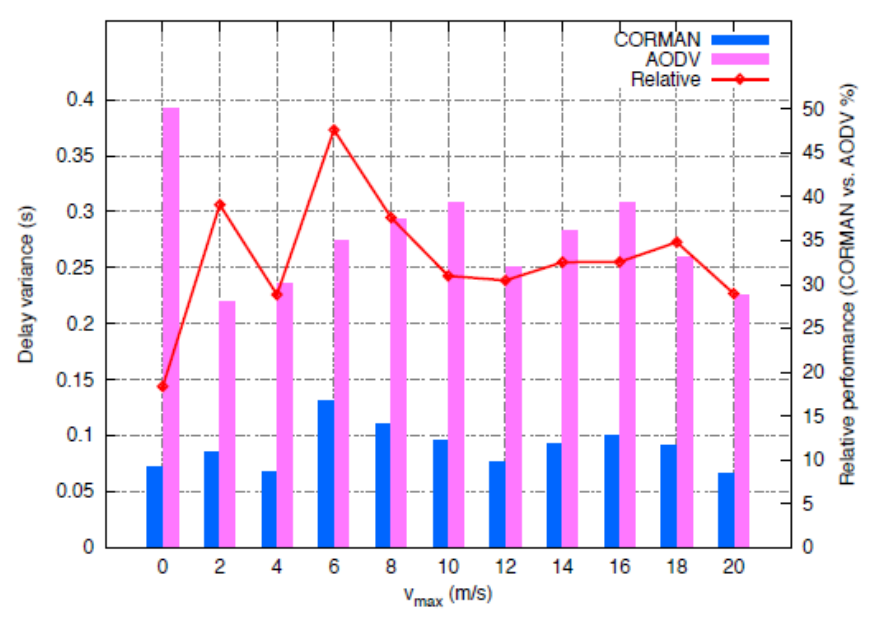

Fig. 13. Delay Jitters. Nodes Mobility.

The delay is plotted versus the network dimension (Fig. 12) to show the performance relation between the CORMAN and AODV. The delay jitter is plotted versus the network dimension (Fig. 13) to show the performance relation between the CORMAN and AODV.

\section{CONCLUSION}

In this paper, a survey of the major types of opportunistic routing is presented. The first type of OR is SDF in 2001, after that ExOR in 2005 then PSR is presented in 2011, after one year a CORMAN is proposed in 2012, and recently in 2015 DPSR is proposed. These types have many drawbacks which can be a rich research area.

\section{REFERENCES}

[1] Chlamtac, M. Conti, and J.-N. Liu, "Mobile ad hoc networking: Imperatives and challenges," Ad Hoc Netw., vol. 1, no. 1, pp. 13-64, Jul. 2003.

[2] R. Rajaraman, "Topology control and routing in ad hoc networks: A survey," ACM SIGACT News, vol. 33, no. 2, pp. 60-73, Jun. 2002.

[3] T. Clausen and P. Jacquet, "Optimized Link State Routing Protocol (OLSR)," RFC 3626, Oct. 2003. [Online]. Available: http://www.ietf.org/rfc/rfc3626.txt

[4] C. E. Perkins and P. Bhagwat, "Highly dynamic Destination-Sequenced Distance-Vector Routing (DSDV) for mobile computers," Comput. Commun.Rev., vol. 24, pp. 234-244, Oct. 1994.

[5] D. B. Johnson, Y.-C. Hu, and D. A. Maltz, "On The Dynamic Source Routing Protocol (DSR) for mobile ad hoc networks for IPv4," RFC 4728, Feb. 2007. [Online]. Available: http://www.ietf.org/rfc/rfc4728.txt

[6] C. E. Perkins and E. M. Royer, "Ad hoc On-Demand Distance Vector (AODV) routing," RFC 3561, Jul. 2003. [Online]. Available: http://www.ietf.org/rfc/rfc3561.txt

[7] P. Larsson, "Selection diversity forwarding in a multihop packet radio networkwith fading channel and capture," ACM Mobile Comput. Commun.Rev., vol. 5, no. 4, pp. 47-54, Oct. 2001.

[8] S. Biswas and R. Morris, "ExOR: Opportunistic multi-hop routing for wireless networks," in Proc. ACM Conf. SIGCOMM, Philadelphia, PA, USA, Aug. 2005, pp. 133-144.

[9] Maleki, Morteza, Karthik Dantu, and Massoud Pedram. "Power-aware source routing protocol for mobile ad hoc networks." Proceedings of the 2002 international symposium on Low power electronics and design. ACM, 2002.

[10] Wang, Zehua, Yuanzhu Chen, and Cheng Li. "CORMAN: A novel cooperative opportunistic routing scheme in mobile ad hoc networks." Selected Areas in Communications, IEEE Journal on 30.2 (2012): 289-296.

[11] Sangheethaa, S., and P. V. Shyamily. "Zone-based Proactive Source Routing Protocol for Ad-hoc Networks." Compusoft 4.1 (2015): 1477.

[12] Haas, Zygmunt J., Marc R. Pearlman, and Prince Samar. "The zone routing protocol (ZRP) for ad hoc networks." (2002). 